\title{
Data Management Best Practices of Complex Socio-technical Systems: A Review of U.S. Mining Safety and Health Management
}

\author{
W. Pratt Rogers*, Michael G. Nelson, Amy Richins and Ashley Hodgson \\ Department of Mining Engineering, University of Utah, Salt Lake City, UT, USA
}

\begin{abstract}
Sociotechnical systems are everywhere in the modern industrial world. Sociotechnical systems describe how humans interact with technology throughout a variety of processes. These systems are prevalent in modern industrial settings, such as operating mines. Safety and health management systems (SHMSs) are complex sociotechnical structures designed with the aim of managing and reducing occupational risk. U.S. mining companies are voluntarily adopting SHMSs to supplement regulatory safety compliance efforts. Like all complex sociotechnical systems there are a wide variety of approaches and adoption philosophies around a SHMS. Many SHMSs suffer from high levels of redundancies, which manifest themselves in diverse ways: duplicate processes, forms, permits, and tracking mechanisms. These redundancies have severe impacts on the overall system and absorb critical culture and leadership "energy". A recent study initiated by the University of Utah and sponsored by the Alpha Foundation is investigating the effectiveness of SHMSs across 15 different U.S. mining sites. A large amount of data has been captured to help characterize elements of SHMSs and correlate their impacts on safety outcomes. Many variables were assessed in the study. Those of importance to this paper are the variables describing the robustness of the sociotechnical aspects of the SHMS. Two such variables are the overall integration of the SHMS within the whole system and how data is managed. Data management in this context deals with the approach to capturing, analyzing, and acting on data within the system. As well, systems are integrated around controlled, data-sharing mechanisms. It has been found that wide variety of approaches has been taken to integrate the SHMS within the governing management philosophy at a given site. A series of case studies is presented that develop some specific best practices for proper data management and integration of SHMSs.
\end{abstract}

Keywords: sociotechnical systems, health and safety management systems, operational excellence, data management

\section{Introduction and Project Background}

Managing occupational health and safety can be done in many different ways, and with many different approaches, but is often based on the host country's regulatory and industrial culture. However, many companies have extended their effort beyond the regulatory requirements of their host nation and have implemented Safety and Health Management Systems. The goal of these efforts is to reduce and eliminate occupational risk. Many health and safety practitioners have a hard time justifying various elements of health and safety management. There is a lack of institutional knowledge that measures and assesses the effectiveness of these Safety and Health Management System (SHMS) elements. To better understand the effectiveness of SHMSs the Alpha Foundation has funded a research project with the University of Utah. The title of the project is "Characterization and Effectiveness of Safety and Health Management Systems in the U.S. Mining Industry." The research team will assess the SHMSs of 15 United States mine sites. The outcome of the project will be a method to characterize the strength of the SHMS elements, assess a culture and leadership index, and finally establish correlations between elemental risk and safety outcomes. The study is currently in progress. However, many important outcomes have already been observed, particularly on the data management practices within an SHMS.

Some general observations of approaches thus far in the research, are: First, many sites have opted for an "anti-system system." This mentality is to reduce the bureaucracy of health and safety management and focus more on the people. Second, other sites have formal systems that are audited by third parties. Third, some sites utilize the corporate SHMS of their international parent company. The wide variety of approaches and their respective successes and failures is important to capture and report. Another observation is that SHMSs and mine sites are complex socio-technical systems. Socio-technical systems include a large number of people interacting with technology through defined work processes, as shown in Figure 1.

The final observation made at this stage of the research project is that proper information and data management is a critical component of any SHMS. No matter the approach adopted, it successfully maintains regulatory and company information about occupational safety events, such as incident investigations, risk assessments, and corrective actions. There is an assortment of approaches to information

* Corresponding Author: W.P. Rogers, pratt.rogers@utah.edu, phone: +1 801-585-3360

Copyright $\odot 2017$ Canamaple Academia Services, http://press.camdemia.ca

DOI: 10.15273/gree.2017.02.016 


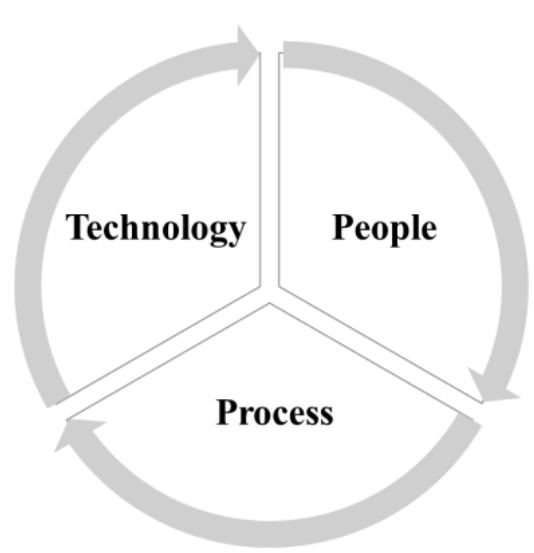

Figure 1. General elements of a socio-technical system.

and data management and it can be a good indicator of the overall health of the system. This statement is the focus of the remaining part of this paper.

To establish context for the statement, the paper will take the following form. First, concepts of socio-technical systems and data management within large-scale mining companies will be reviewed. This discussion will be followed by the approach used to determine the elemental risk of an SHMS and how data management will be an important indicator. Some initial findings and best practices for data management will then be given. Lastly, the paper will conclude with the next steps and a discussion on what to expect from the remaining aspects of the study.

\section{Concept Introduction: Socio-technical Systems and Data Management}

Safety and health management systems have been studied for years (International Organization for Standardization 2017, Robson et al 2007, Arcocena and Nunez 2010). Additionally, the complexity of risk management in occupational health and safety is representative of a socio-technical system (Rasmussen 1997, Qureshi 2007, Mohaghegh and Mosleh 2009). Data, knowledge, and information management within socio-technical systems has been well researched (Bhatt 2001, Herbsleb 2007, Alter 2013). This paper hopes to fill in the gaps on the facilitating role of data management within an SHMS. A quick review of socio-technical systems is given, followed by some data management considerations.

\subsection{Sociotechnical systems}

Sociotechnical systems are changing the world from standard systems to systems that incorporate social human factors with an infrastructure that supports a company's specific objectives (Tong 2016). This departs from older systems that were designed with only technological factors. There is an ever-increasing need to adapt and improve the capacity and effectiveness of systems with sociotechnical considerations (Nelson 2016).

Socio-technical systems contain the complex work processes of humans and technology. They are important because they consider complex human factors (Wickens et al 2013). Human factors analysis is the study of factors that affect human performance in complex systems. As a discipline, human factors analysis, is focused on understanding interactions between people and other elements of complex systems, such as hardware, software, the environment (Keating et al 2001). An important element of the Human Factors Engineering (HFE) perspective is the conceptualization of any system as a socio-technical system. A socio-technical system can be characterized by its components (Drews 2013). According to Drews the five elements of a socio-technical system are: people, work, work organization, equipment, and environment.

Most modern SHMSs are complex socio-technical systems (Rasmussen 1997). Additionally, big data analysis and data accountability are increasingly being considered as key elements of socio-technical analysis (Boyd and Crawford 2012, Pretschner and Waltl 2016). SHMSs can create large numbers of procedures and can be dependent upon knowledge discovery within complex data sets. Therefore, data management is an important aspect of the success of an SHMS.

\subsection{Data management defined}

Modern industrial practices and processes are transitioning from an "information era" into a "digital era" (Hilbert and Lopez 2012). The difference between these eras is the proliferation of digital technologies that generate data. More data has been created by these digital technologies in the past two years than in many previous decades (SINTEF 2013). Industries are generating more data than ever before and at an increasing pace.

Information Systems (IS) have matured rapidly over the past decades and the Management of Information Systems (MIS) industry has developed IS success models that assist in understanding the frameworks that lead to successful deployments (Nelson et al 2005). What's more, successful deployments are the outcome and driven by use of the systems or utilization of data (Bradley et al 2006) and (Rogers 2015). Because SHMSs are increasingly dependent upon technology and systems, data management is critical to the successful adoption and sustainability of the system. Figure 2 illustrates a process of data management to facilitate analytics in a complex sociotechnical system.

As shown in Figure 2, the first step in data management is data input. In any system there are inputs. These can come from a variety of sources and approaches, both contemporary and new age, such as the internet of things (IOT) (Hilbert and Lopez 2012). The next step is to capture the data in a defined location and process. Complex systems have many sources of data that is stored in databases. A key element of any data management approach is a data integration platform (Dessureault and Scoble 2003). Data integration leads to greater insight and understanding of the system (Dessureault 2002). Malcom Gladwell (2007) states that "The key to good decision making is not knowledge. It is understanding. We are swimming in the former. We are desperately lacking in the latter." When decision makers are deriving understanding from their system of processes, they are taking a more informed and appropriate action. This is dependent upon a formal business intelligence hierarchy, which provides the right data to the right person at the right time (Vercellis 2011). The most recognizable step in data management, big data 
analysis, seems to be the most misunderstood (Boyd and Crawford 2012). Essentially big data analysis facilitates knowledge discovery by looking for patterns not perceived by humans. To achieve this level of analysis the data set must be large and accurate. In most cases, an SHMS requires just a business intelligence analytical layer.

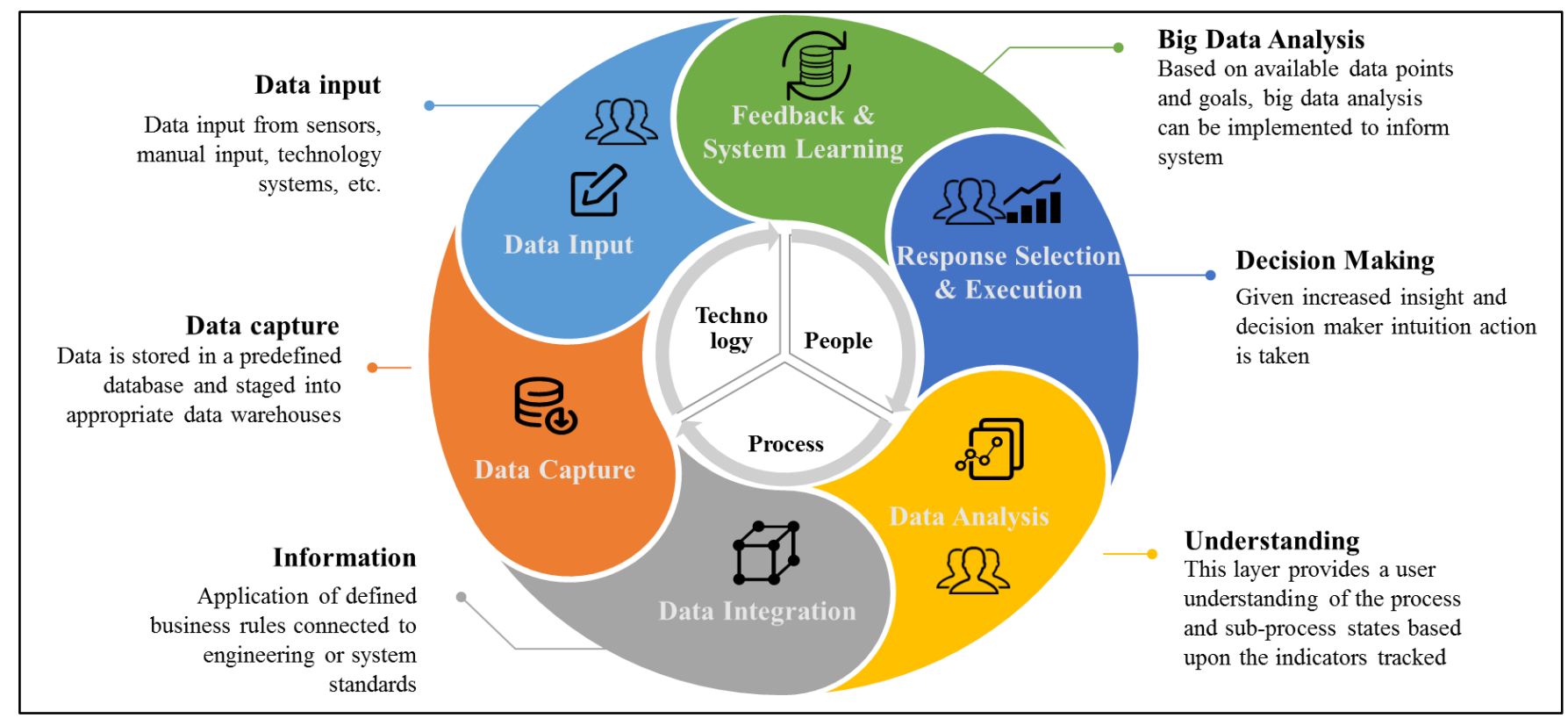

Figure 2. Data analysis and management process.

\section{SHMS Assessments and Data Management Practices}

Socio-technical systems consider the human factors and enabling work processes and technologies that facilitate efficient operating parameters within a system. Data management practices can facilitate understanding of the work processes of the system. Human factors and data management are socio-technical enablers of any system, and can therefore be considered key elements of SHMS assessments and indicators of the overall health of the system. Thus researchers should treat the data and information management within an SHMS as a significant variable in assessing the effectiveness of the overall system.

\subsection{SHMS assessments}

One outcome of the authors' research project is assessing the effectiveness of the elements within an SHMS. Table 1 shows a list of common SHMS elements. A given system may have more or fewer elements, but those shown are typical.

Through a series of interviews and reviews of a particular companies, SHMS policies and procedures, the researchers determine an elemental risk factor to each level. Elemental risk within an SHMS describes the level of consequence and likelihood that the element will have on negatively impacting the effectiveness of the companies SHMS.

Each element of a system consists of an input, output, and process, as shown in Figure 3.
Table 1. SHMS elements assessed.

\begin{tabular}{|c|c|}
\hline Element Name & Elemental Risk \\
\hline $\begin{array}{l}\text { Risk Management Fatality } \\
\text { Prevention } \\
\end{array}$ & 0-15 Rating \\
\hline Behavior Based Safety & 0-15 Rating \\
\hline Training and Competence & 0-15 Rating \\
\hline Emergency Management & 0-15 Rating \\
\hline Work Procedures and Permits & 0-15 Rating \\
\hline Occupational Health & 0-15 Rating \\
\hline Incident and Reporting and Permits & 0-15 Rating \\
\hline $\begin{array}{c}\text { Documentation and Information } \\
\text { Mgmt. }\end{array}$ & 0-15 Rating \\
\hline Change Management & 0-15 Rating \\
\hline Engineering and Construction & 0-15 Rating \\
\hline Contractor Mgmt. and Purchasing & 0-15 Rating \\
\hline Responsibility and Accountability & 0-15 Rating \\
\hline Leadership Development & 0-15 Rating \\
\hline Established Vision and Strategy & 0-15 Rating \\
\hline Collaboration and Communication & 0-15 Rating \\
\hline Culture Enhancement & 0-15 Rating \\
\hline
\end{tabular}




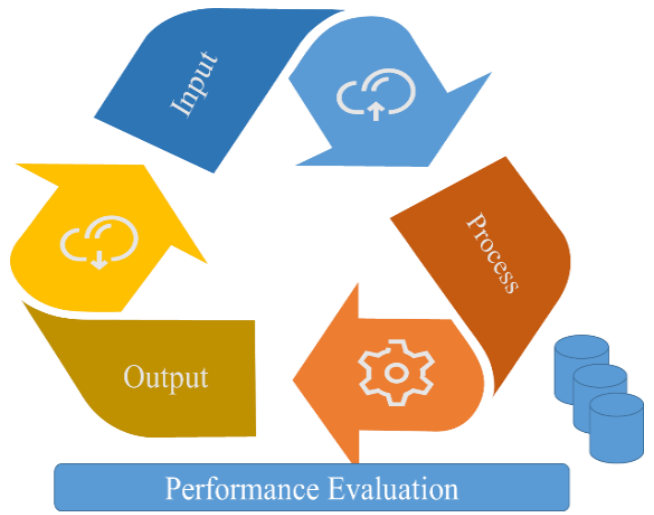

Figure 3. General characteristics of safety and health management system elements.

An additional characteristic of an SHMS element is a performance evaluation. The performance of each element creates a need to manage data to some degree or another. Consider a formal incident investigation. After an occupational incident occurs, a formal investigation ensues. This investigation will follow a certain procedure and process. Inputs will be gathered, and corrective actions will be reviewed. The details of the incident and the corrective action must be maintained and stored. These can be living and breathing data sets that are continually augmented. Therefore, managing the data created by a given element is critical to the effectiveness of that element. As shown in Table 1, there typically 15 elements within an SHMS. Data management within an SHMS can be complicated. The next section gives specifics of common practices within an SHMS.

\subsection{Data management practices within a SHMS}

Section 2.2 provided general steps considered in data management and the analytical process. SHMSs follow a similar management process with some additional items. In the context of an SHMS some common items tracked are:

- Document control

- Standard operating procedures (SOPs)

- Work permits

- Area inspection and audits

- Observations

- Regulatory compliance documents

- Proof of training

- Occupational health and safety event tracking

- Incidents, near-misses, non-reportable incidents

- Inspections, audits, etc.

- Risk assessment and registers

- Performance metrics

- Reports

- Performance feedback and improvement

- Business intelligence analysis

- Trending

- Root Cause Analysis

- Knowledge discovery (BDA)

- Histograms

While on site, the research team tracks down these data points, reports, documents, and processes. The degree to which they are organized, accurate, and systematic is indicative of the elemental risk of the SHMS. In good management of occupational safety and health, there are many moving parts that go beyond just the safety department. The next section will note some key findings the research team made during the initial assessments.

\section{Key Findings and Discussion}

Data management practices varied from site to site. Companies used file servers, cloud shared servers, Microsoft Excel, Access databases, enterprise systems, tablets, and formal Environmental, Health and Safety (EHS) web applications. Some companies also created homegrown software or used tools developed by outside consultants. A common observation was that more systematic the data management contributed to a more organized the overall SHMS. Table 2 provides a review of the sites and brief overview of the data management ecosystem.

At the time of writing this paper, not all of the sites have been visited. However, there are some items to discuss from the initial surveyed sites. Table 2 considers four main data management groups. The first is occupational health and safety events such as incident investigations, risk assessments, and near misses. The next is document control, which considers how policies, SOPs, training, and regulatory files are maintained and controlled. Next, the business metrics group of data management is considered. Business metrics report on summarized and consolidated data on the work processes and occupational outcomes of the site. The last group is analysis. This set considers the activities the site is doing to dive deeper into the data of the SHMS and derive understanding. Some general observations and best practices are discussed.

Table 2. Description of data management practices of sites included in study.

\begin{tabular}{|c|c|c|c|c|}
\hline Site & $\begin{array}{c}\text { Occupational } \\
\text { H\&S Events } \\
\end{array}$ & $\begin{array}{c}\text { Document } \\
\text { Control }\end{array}$ & Metrics & Analysis \\
\hline $\begin{array}{c}\text { Site } \\
1\end{array}$ & $\begin{array}{c}\text { EHS } \\
\text { Enterprise } \\
\text { Software }\end{array}$ & $\begin{array}{l}\text { Shared } \\
\text { Server }\end{array}$ & Scorecards & $\begin{array}{c}\text { None } \\
\text { observed }\end{array}$ \\
\hline $\begin{array}{c}\text { Site } \\
2\end{array}$ & Excel & $\begin{array}{l}\text { Shared } \\
\text { Server }\end{array}$ & None & $\begin{array}{c}\text { None } \\
\text { observed }\end{array}$ \\
\hline $\begin{array}{c}\text { Site } \\
3 \\
\end{array}$ & Excel & $\begin{array}{l}\text { Shared } \\
\text { Server }\end{array}$ & None & $\begin{array}{c}\text { None } \\
\text { observed }\end{array}$ \\
\hline $\begin{array}{c}\text { Site } \\
4\end{array}$ & $\begin{array}{c}\text { Custom Web } \\
\text { app }\end{array}$ & $\begin{array}{c}\text { Shared } \\
\text { Server / } \\
\text { Files }\end{array}$ & $\begin{array}{l}\text { Daily } \\
\text { reports }\end{array}$ & $\begin{array}{c}\text { Root cause } \\
\text { analysis }\end{array}$ \\
\hline $\begin{array}{l}\text { Site } \\
5\end{array}$ & $\begin{array}{c}\text { Enterprise } \\
\text { resource } \\
\text { planning } \\
(\mathrm{ERP})\end{array}$ & $\begin{array}{c}\text { Shared } \\
\text { Server / } \\
\text { Files }\end{array}$ & $\begin{array}{l}\text { Daily } \\
\text { reports }\end{array}$ & $\begin{array}{c}\text { Root cause } \\
\text { analysis }\end{array}$ \\
\hline
\end{tabular}

Document control is always a challenge. Tools like Microsoft's SharePoint and custom-shared file servers help facilitate this. However, many safety managers had difficulty in finding SHMS documents during the interviews. Some companies maintained folders of printed papers. 
Organizations that had a file server and rules on folder structures maintained better document control.

Data capture of occupational health and safety events varied from company to company. Two companies had an enterprise-wide environmental, health, and safety (EHS) software package. This web application allowed safety personnel to track incidents, corrective actions, audits, and many other occupational health and safety events. However, these companies struggled with replacing old Access databases, Excel sheets, and existing reports. This caused significant redundancies in their SHMS systems. A significant cause of this deficiency was a lack of training on the software for the safety professionals.

A few companies deployed mobile tablets to allow front line workers to fill out inspections and reports. Capturing data with tablets and eliminating paper is a great way to eliminate redundant entry of SHMS data. Companies are continuing to explore the role of IOT and additional sensors for occupational health.

Business metrics and analyses were challenging for almost all the mine sites visited. It was common for the team to observe safety managers and their support team spending a full day each week developing manual reports. This time pulled the team away from key health and safety activities like audits and inspections. A few sites were able to do rootcause analyses and trending of their occupational health and safety events. One company had recently deployed an EHS platform and was in the middle of transitioning from an Excel and Access database system.

Data management was the critical element for all sites visited. The safety departments were capable of developing accurate and appropriate work processes and procedures. However, the team observed many safety managers act as glorified data clerks and spending a great deal of time preparing reports.

The widest variety of data management approaches seen at the sites studied was on document control. This seemed to be the common challenge for all the companies. Some companies maintain rigid policies for their SharePoint file server. Other companies had their IT department set up a shared file server. Documents such as SOPs or company SHMS policies were maintained in a folder structure with no formal folder structure. A few safety managers had a difficult time producing specific polices on an SHMS element when asked.

\section{Conclusions}

SHMS science and thought are continually advancing, as evidenced by the upcoming release of ISO 43.001 (International Organization for Standardization 2017). SHMSs must adapt with the evolution of enabling technology advances such as IOT, business intelligence, and mobile computing. Mining companies must invest in the technology and not just the thought processes of SHMSs. This investment should consider the process in Figure 2. Many organizations are acting as early adopters of this technology. Technology and newly prescribed processes can be developed and installed with relative ease. However, technology and process implementation must consider human factors and ease of use. SHMS experts and mining companies must not confuse installation with implementation. Social acceptance is critical for true implementation to occur. With any deployment, human factors engineering within complex socio-technical systems must be considered. The goal should be focusing on breaking down discipline and organizational silos.

Data management is a big undertaking that requires much thought and a wide range of expertise. Document control strategy and business metrics are two very different areas. Health and safety management professionals need the expertise to manage these areas to meet business objectives. To fully achieve the benefits of an SHMS, mining companies must have organized and systematic data management procedures.

\section{Next Steps}

The research team will continue to proceed with the study on assessing the SHMS effectiveness at United States based mines. As the SHMSs are characterized and implementations are assessed, an elemental risk factor will be given. The team will establish correlations between elemental risk and safety outcomes such as reportable incidents. These correlations will provide the health and safety practitioners valuable information to cite and justify elements of a proposed system. Future areas of research can extend to establish causation factors of SHMS elements. These causations could provide insight to predict safety outcomes.

Data management best practices will continually evolve with changing technology. The industrial internet of things (IIOT) will extend the digital reach of all SHMSs. Success of these tools will be dependent upon consideration of human factors and work practices.

\section{Acknowledgements}

This project was made possible by the financial support of the Alpha Foundation for the Improvement of Mine Safety and Health, Inc., through grant number AFC 215-30. The authors also wish to thank and mention the un-named companies that allowed researchers on site to conduct the surveys and audits. Their participation made this research project possible.

\section{References}

Alter, S., 2013. Work system theory: overview of core concepts, extensions and challenges for the future. Journal of the Association for Information Systems, 14(2): 72.

Arcocena, P., and I. Nunez, 2010. An empirical analysis of the effectiveness of occupational health and safety management systems in SMEs. International Small Business Journal, 28(4): 398 - 419.

Bhatt, G., 2001. Knowledge management in organizations: examining the interaction between technologies, techniques, and people. Journal of Knowledge Management, 5(1): 68 - 75. 
Boyd, D., and K. Crawford, 2012. Critical questions for big data: Provocations for a cultural, technological, and scholarly phenomenon. Information, Communication \& Society, 15(5): 662 - 679.

Bradley, R.V., J.L. Pridmore and T.A. Byrd, 2006. Information systems success in the context of difference corporate culture types: An empirical investigation. Journal of Management Information Systems, 23(2): 267 $-294$.

Dessureault, S., 2002. Tactical mine management. Retrieved from The University of British Columbia. http://hdl.handle.net/2429/12942.

Dessureault, S., and M. Scoble, 2003. Data infrastructure for a tactical mine management system. Mine Technology, 112: $73-85$.

Drews, F.A., 2013. Human factors in critical care medical environments. Reviews of Human Factors \& Ergonomics, 2013, 8(8): 103 - 148.

Gladwell, M., 2007. Blink: The power of thinking without thinking. Black Bay Books.

Herbsleb, J., 2007. Global software engineering: The future of socio-technical coordination. Future of Software Engineering, 2007: 188 - 198.

Hilbert, M., and P. Lopez, 2012. How to measure the worlds technological capacity to communicate, store and compute information? Part I: results and scope. International Journal of Communication, 6: 956 - 979.

International Organization for Standardization, 2017. Retrieved from ISO 45001 - occupational health and safety. https://www.iso.org/iso-45001-occupational-healthand-safety.html.

Keating, K., D. Fernandez, and J. Kauffmann, 2001. A methodology for analysis of the complex sociotechnical processes. Business Process Management Journal, 7(1): $33-50$.

Mohaghegh, Z., and A. Mosleh, 2009. Incorporating organizational factors into probabilistic risk assessment of complex socio-technical systems: Principles and theoretical foundations. Safety Science, 47(8): 1139 1158 .
Nelson, P.P. 2016. A framework for the future of urban underground engineering. Tunneling and Underground Space Technology, 55: 32 - 39.

Nelson, R.R., P.A. Todd and B.H. Wixom, 2005. Antecedents of information and system quality: An empirical examination within the context of data warehousing. Journal of Management Information Systems, 21(4): 199 - 235.

Pretschner, A. and B. Waltl. 2016. Data accountability in socio - technical systems. Proceedings of 17 th International Conference of Enterprise, BusinessProcess and Information Systems Modeling. Ljubljana, Slovenia, 335 - 348.

Qureshi, Z.H. 2007. A review of accident modelling approaches for complex socio-technical systems. Twelfth Australian Workshop on Safety Critical Systems \& Software \& Safety-related Programmable Systems, 47 - 59.

Rasmussen, J. 1997. Risk management in a dynamic society: a modeling problem. Safety Science, 27(2): 183 - 213.

Robson, L.S., K. Cullen, A. Bielecky, C. Severin, P. Bigelow, E. Irvin, and Q. Mahood, 2007. The effectiveness of occupational health and safety management system interventions: a systematic review. Safety Science, 45(3): $329-353$

Rogers, W.P. 2015. Formal assessment and measurement of data utilization and value for mines. Ph.D. dissertation, University of Arizona, AZ.

SINTEF, 2013. ScienceDaily. Big Data, for better or worse: $90 \%$ of world's data generated over last two years. https://www.sciencedaily.com/releases/2013/05/130522 085217.htm.

Tong, L. 2016. Holistic security requirements engineering for socio-technical systems, University of Trento, Trento, Italy.

Vercellis, C. 2011. Business intelligence: data mining and optimization for decision making. John Wiley and Sons, 110(493): 376.

Wickens, C.D., J.G. Hollands, S. Banbury and R. Parasuraman, 2013. Engineering Psychology and Human Performance, 4th Ed. Boston: Pearson. 